\author{
H. Witała • J. Golak • R. Skibiński • K. Topolnicki • \\ H. Kamada • E. Epelbaum • W. Glöckle • H. Krebs • \\ W. N. Polyzou · A. Nogga
}

\title{
Calculations of Three-Nucleon Reactions
}

Received: 24 September 2012 / Accepted: 18 December 2012 / Published online: 8 January 2013

(C) The Author(s) 2013. This article is published with open access at Springerlink.com

\begin{abstract}
Faddeev calculations using the chiral three-nucleon force in next-to-next-to-next-to-leading-order show that this force is too weak to provide an explanation for the low-energy $A_{y}$ puzzle. The large discrepancy between data and theory for the neutron-neutron quasi-free-scattering cross section in low energy neutrondeuteron breakup requires a modification of the ${ }^{1} S_{0}$ neutron-neutron force. We discuss the consequences that a bound ${ }^{1} S_{0}$ state of two neutrons has on neutron-deuteron scattering observables. At higher energies we compare the solutions of the non-relativistic three-nucleon Faddeev equations with three-nucleon force included to the solutions of its Poincaré invariant version.
\end{abstract}

\section{Introduction}

Recent progress in the construction of chiral nucleon-nucleon (NN) and three-nucleon forces (3NF) allows to test chiral dynamics in $3 \mathrm{~N}$ reactions up to the next-to-next-to-next-to-leading-order $\left(\mathrm{N}^{3} \mathrm{LO}\right)$ of the chiral expansion. It provides also an opportunity to test if consistent two- and three-nucleon forces are able to explain the low-energy $A_{y}$ puzzle.

The large disagreement between theory and data for the neutron-neutron quasi-free scattering (nn QFS) cross section in low energy neutron-deuteron (nd) breakup reaction indicates the possibility that two neutrons can form a bound state when interacting in a ${ }^{1} S_{0}$ state. We discuss consequences of the existence of a dineutron on observables in nd reactions.

The study of nucleon-deuteron (Nd) elastic and breakup processes revealed that at higher energies there are cases where the non-relativistic description based on $\mathrm{NN}$ interactions only is insufficient to explain the data.

On 1 August 2012 Walter Glöckle passed away. We dedicate this paper to Walter, who was a great friend and collaborator.

H. Witała $(\varangle) \cdot$ J. Golak · R. Skibiński · K. Topolnicki

M. Smoluchowski Institute of Physics, Jagiellonian University, 30059 Kraków, Poland

E-mail: witala@if.uj.edu.pl; henryk.witala@uj.edu.pl

H. Kamada

Department of Physics, Faculty of Engineering, Kyushu Institute of Technology, Kitakyushu 804-8550, Japan

E. Epelbaum · W. Glöckle $\cdot$ H. Krebs

Institut für theoretische Physik II, Ruhr-Universität Bochum, 44780 Bochum, Germany

W. N. Polyzou

Department of Physics and Astronomy, The University of Iowa, Iowa City, IA 52242, USA

A. Nogga

Forschungszentrum Jülich, Institut für Kernphysik, Institute for Advanced Simulation and Jülich Center for Hadron Physics, 52425 Jülich, Germany 


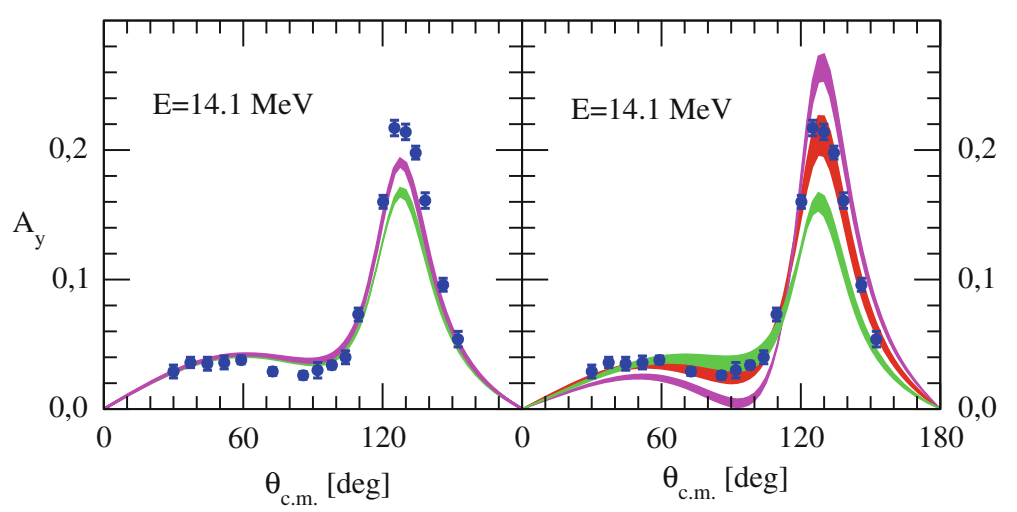

Fig. 1 (color online) The neutron analyzing power $A_{y}$ in elastic nd scattering. In the left column the light shaded (green) and dark shaded (magenta) bands show predictions of realistic NN potentials (AV18, CD Bonn, Nijm1 and Nijm2) alone or combined with TM 3NF, respectively. In the right column the magenta (upper), red (middle) and green (low) bands show predictions of NLO, $\mathrm{N}^{2} \mathrm{LO}$, and $\mathrm{N}^{3} \mathrm{LO}$ chiral NN potentials, respectively. The nd data (full circles) are from [6]

These discrepancies generally increase with energy. Only in some cases does the inclusion of certain types of $3 \mathrm{~N}$ forces lead to an improvement. We discuss effects of relativity on higher-energy Nd elastic scattering and breakup observables.

\section{$2 A_{y}$ Puzzle and the $\mathrm{N}^{3} \mathrm{LO}$ Chiral Three-Nucleon Force}

In order to describe the $2 \mathrm{~N}$ system with the same high precision as provided by standard semi-phenomenological $\mathrm{NN}$ potentials one needs to go to $\mathrm{N}^{3} \mathrm{LO}$ in chiral expansion $[1,2]$. In the following, results of $3 \mathrm{~N}$ Faddeev calculations based on five versions of chiral $\mathrm{N}^{3} \mathrm{LO}$ potentials, which use different cut-off's for the LippmannSchwinger equation and spectral function regularization [1] and which equally well describe the $2 \mathrm{~N}$ system, will be presented. In that order of the chiral expansion six topologies contribute to the $3 \mathrm{NF}: 2 \pi$-exchange, $2 \pi-1 \pi$-exchange, ring, $1 \pi$-exchange-contact, $2 \pi$-exchange-contact and a purely contact term. In addition, there are also leading relativistic corrections. The first three topologies belong to long-range contributions [3], while others are of short-range character [4]. These terms do not involve any unknown low-energy constants and the full $\mathrm{N}^{3} \mathrm{LO} 3 \mathrm{NF}$ depends on two parameters, $\mathrm{D}$ and $\mathrm{E}$, coming with the $1 \pi$-exchange-contact and the purely contact term, respectively. A recently developed efficient method of partial wave-decomposition [5] allowed us to apply the $\mathrm{N}^{3} \mathrm{LO} 3 \mathrm{NF}$ in $3 \mathrm{~N}$ Faddeev calculations. First results presented in the following were obtained without the short-range $2 \pi$-exchange-contact term and leading relativistic corrections in that $3 \mathrm{NF}$. In the left column of Fig. 1 the $A_{y}$ puzzle is exemplified for nd data taken at $14.1 \mathrm{MeV}$. High-precision semiphenomenological NN potentials (light shaded band) cannot describe the data and including the $2 \pi$-exchange Tucson-Melbourne (TM) 3NF (dark shaded band) only partially fills out the discrepancy in the maximum of $A_{y}$. Taking the next-to-leading order (NLO) chiral NN potential overestimates the data for $A_{y}$ (upper band in the right column of Fig. 1), while next-to-next-to-leading order $\left(\mathrm{N}^{2} \mathrm{LO}\right)$ potentials describe the $A_{y}$ data quite well (middle band in the right column of Fig. 1). Such behavior can be traced back to the large sensitivity of $A_{y}$ to the ${ }^{3} P_{j} \mathrm{NN}$ force components and to a poor description, especially for ${ }^{3} P_{2}$, of the experimental phase-shifts by the NLO and $\mathrm{N}^{2} \mathrm{LO}$ chiral potentials [1]. Only with the $\mathrm{N}^{3} \mathrm{LO} \mathrm{NN}$ potentials is the $A_{y}$ puzzle again regained (lower band in the right column of Fig. 1) and predictions for $A_{y}$ become similar to those obtained with semi-phenomenological potentials.

The chiral $\mathrm{N}^{3} \mathrm{LO} 3 \mathrm{NF}$ is not able to explain the $A_{y}$ puzzle (see Fig. 2). The effect of that force is practically negligible and it slightly lowers the $A_{y}$ maximum. A resolution of the $A_{y}$ puzzle must thus be due to either the $\mathrm{N}^{4} \mathrm{LO}$ chiral $3 \mathrm{NF}$ [7] or/and an incorrect knowledge of the low-energy ${ }^{3} P_{j}$ NN phase-shifts.

\section{The Dineutron and Its Influence on nd Observables}

Cross sections for the symmetric-space-star (SST) and quasi-free-scattering (QFS) configurations of the nd breakup are extremely stable with respect to the underlying dynamics. Different potentials, alone or combined 


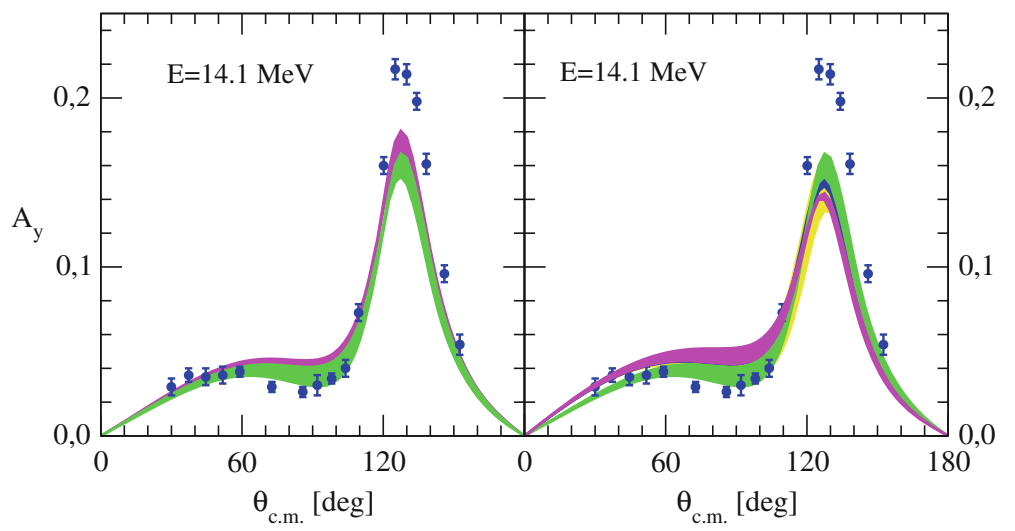

Fig. 2 (color online) The neutron analyzing power $A_{y}$ in elastic nd scattering. In the left column the light shaded (green) band shows predictions of $\mathrm{N}^{3} \mathrm{LO}$ chiral NN potentials alone and the dark shaded (magenta) band when they are combined with $\mathrm{N}^{2} \mathrm{LO}$ chiral $3 \mathrm{NF}$. In the right column the $\mathrm{N}^{3} \mathrm{LO}$ chiral forces (green band) are combined with $\mathrm{N}^{3} \mathrm{LO} 3 \mathrm{NF}$ composed of $1 \pi$-exchange-contact and purely contact terms supplemented with long-range terms: $2 \pi$-exchange (yellow band), $2 \pi$ - and $2 \pi-1 \pi$-exchange (blue band), and $2 \pi$ - and $2 \pi-1 \pi$-exchange and ring (magenta band). The nd data (full circles) are from [6]

with standard $3 \mathrm{~N}$ forces, provide practically the same SST and QFS cross sections. Also, the chiral $\mathrm{N}^{3} \mathrm{LO}$ $3 \mathrm{NF}$ is no exception and cannot explain the discrepancy with the data found for the SST configuration [8]. At low energies the cross sections in the SST and QFS configurations are dominated by S-waves. For the SST configuration the largest contribution to the cross section comes from ${ }^{3} S_{1}$ while for neutron-neutron (nn) QFS the ${ }^{1} S_{0}$ dominates. Neglecting rescatterings the QFS configuration resembles free NN scattering. For free, low-energy neutron-proton (np) scattering one expects contributions from ${ }^{1} S_{0} \mathrm{np}$ and ${ }^{3} S_{1}$ forces. For free $\mathrm{nn}$ scattering only the ${ }^{1} S_{0} \mathrm{nn}$ is allowed. That implies that QFS nn would be a powerful tool to study the $\mathrm{nn}$ interaction.

The measurement of QFS np cross sections have shown good agreement of data with theory [9], confirming thus good knowledge of the np force. For nn QFS it was found that theory underestimates the data by $\approx 20 \%$ [9]. The large stability of the QFS cross sections to the underlying dynamics, implies that the present day ${ }^{1} S_{0} \mathrm{nn}$ interaction is probably incorrect. Modifications of the ${ }^{1} S_{0} \mathrm{nn}$ force by multiplying its matrix elements by a factor $\lambda$ lead to large changes of the nn QFS cross sections, leaving the np ones practically unchanged [10-12]. To remove the discrepancy found in experiment for nn QFS one needs to increase $\lambda$ by about $8 \%$. Such increased strength of the ${ }^{1} S_{0} \mathrm{nn}$ force leads to a nearly bound ${ }^{1} S_{0}$ state of two neutrons $[11,12]$. That raises the question to what extent is the existence of ${ }^{1} S_{0}$ dineutron compatible with available nd data. It turns out that the total nd cross section data, total nd elastic scattering cross section and total nd breakup cross section seem not to exclude two neutrons being bound with $\mathrm{a} \approx-100 \mathrm{keV}$ binding energy [12]. The dineutron influences the nd elastic scattering angular distribution only at forward angles, changing the slope of the cross section. No reliable data at these angles are available [12]. The strongest argument against the dineutron is provided by four measured nn final-state interaction (FSI) configurations [13]. Their analysis gave consistent negative values for the $\mathrm{nn}$ scattering length. It seems that with a positive scattering length one would get nn scattering length values which are configuration dependent. Changing to positive nn scattering lengths reduces drastically the magnitude of the FSI peak at large proton energy in the spectra of protons from incomplete nd breakup. Integrating the experimental peak provides an angular distribution for $n+d \rightarrow p+$ dineutron transition. Comparing it to theoretical values excludes binding energies for dineutron larger in magnitude than $\approx 100 \mathrm{keV}$ (see Fig. 3).

The most favorable conditions to detect the dineutron would exist when two neutrons mostly occupy the ${ }^{1} S_{0}$ state. Such a situation is provided by the ${ }^{3} \mathrm{H}$ nucleus and the $\gamma\left({ }^{3} \mathrm{H}, \mathrm{p}\right) n n$ reaction seems to be advantageous in searching for the dineutron. The spectra of outgoing protons in that reaction are strongly distorted by the existence of the dineutron (see Fig. 4) and in addition to that distortion a peak corresponding to $\gamma+{ }^{3} \mathrm{H} \rightarrow p+$ dineutron transition should appear at largest outgoing proton energies. The magnitude of that peak is determined by the angular distribution for that transition.

The existence of the dineutron does not directly explain the discrepancy in the cross sections in SST configuration. Taking $\lambda$ values for which a dineutron exists even increases that discrepancy [12]. This does not 


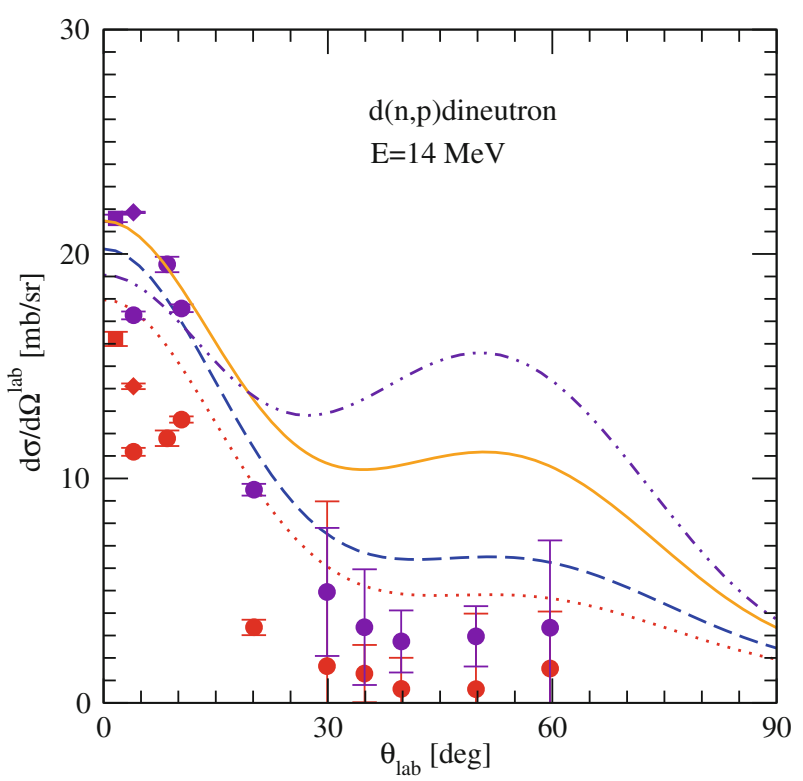

Fig. 3 (color online) Lab. angular distribution for d(n,p)dineutron reaction at $E=14 \mathrm{MeV}$ incoming neutron lab. energy. Different curves correspond to different factor $\lambda$ by which the ${ }^{1} S_{0}$ nn matrix element of the CD Bonn potential was multiplied in order to produce the ${ }^{1} S_{0}$ dineutron. The dotted (red) curve corresponds to $\lambda=1.18$ and the dineutron binding energy $\epsilon_{n n}=-80 \mathrm{keV}$. The dashed (blue) to $\lambda=1.21$ and $\epsilon_{n n}=-144 \mathrm{keV}$, the solid (orange) to $\lambda=1.3$ and $\epsilon_{n n}=-441 \mathrm{keV}$, and the dashed-double-dotted (indigo) to $\lambda=1.4$ and $\epsilon_{n n}=-939 \mathrm{keV}$. The red and violet full circles, squares, and rhombus result from integration of the FSI peak in spectra of outgoing protons from incomplete $d(n, p)$ nn breakup from refs. [14-16], respectively

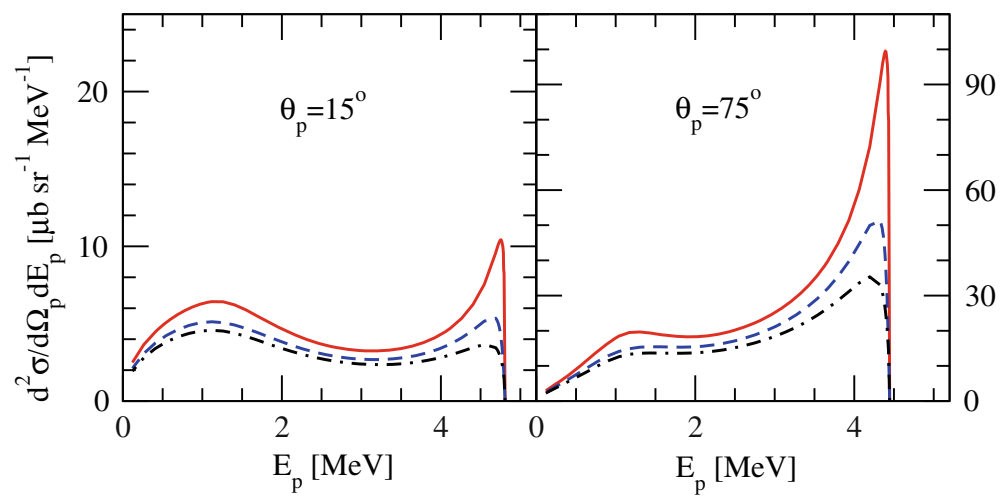

Fig. 4 (color online) The energy spectra of outgoing protons from reaction $\gamma\left({ }^{3} H, p\right) n n$ at $E_{\gamma}=15 \mathrm{MeV}$. The (red) solid curve is based on AV18 potential and standard meson-exchange currents [17]. The (blue) dashed and (black) dashed-dotted curve result when ${ }^{1} S_{0} \mathrm{nn}$ force of AV18 potential is multiplied with factor $\lambda=1.16$ and 1.22 , leading to binding energy of dineutron $\epsilon_{n n}=-108 \mathrm{keV}$ and $-323 \mathrm{keV}$, respectively

exclude, however, the possibility to explain that discrepancy by contributions from secondary reactions, which are possible when the dineutron exists.

\section{Relativistic Effects in 3N Continuum}

At incoming nucleon energies above $\approx 100 \mathrm{MeV}$ clear discrepancies between theory and data, e.g. in the $\mathrm{Nd}$ elastic scattering angular distribution and the nd total cross section, appear, even when a $2 \pi$-exchange $3 \mathrm{NF}$ is included in the calculations. To find out if additional 3NF components, which become active at higher energies, are responsible for these discrepancies, the magnitude of relativistic effects in $3 \mathrm{~N}$ continuum must be determined. 


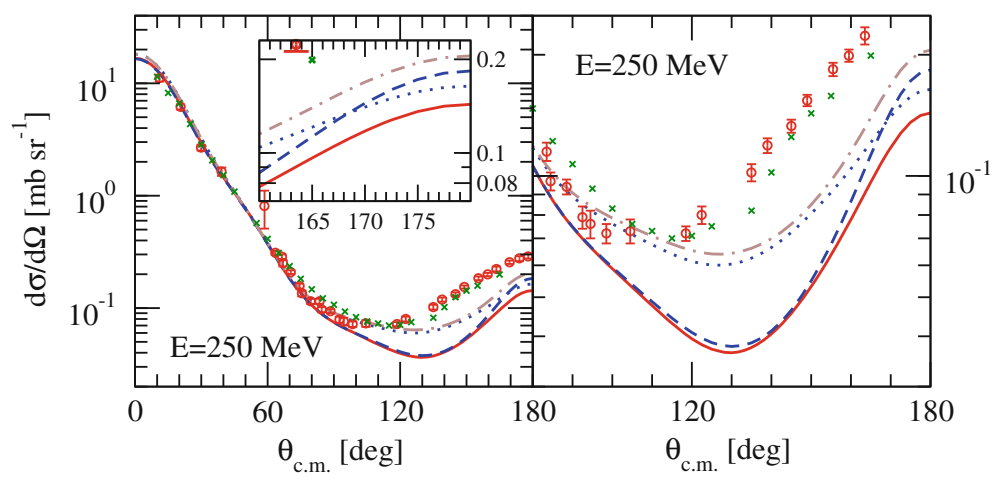

Fig. 5 (color online) The elastic nd scattering angular distributions at the incoming neutron lab. energy $E=250 \mathrm{MeV}$. The solid (red) and dotted (blue) lines are results of the non-relativistic Faddeev calculations with the CD Bonn potential alone and combined with the TM 3NF, respectively. The relativistic predictions based on the CD Bonn potential without Wigner spin rotations are shown by the dashed (blue) lines. The dashed-dotted (brown) lines show results of relativistic calculations with the TM 3NF included. The pd data (x-es) are from ref. [22] and nd data (circles) from ref. [23]. The inset and right figure display details of the cross sections in specific angular ranges

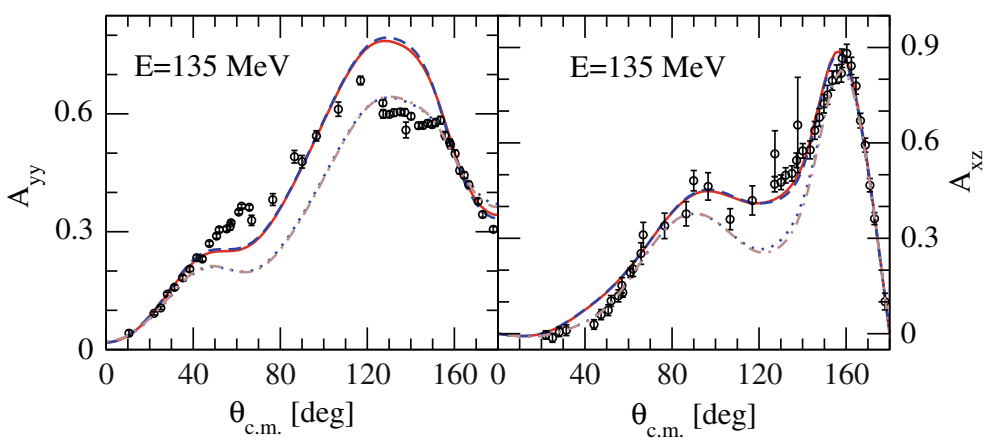

Fig. 6 (color online) The tensor analyzing powers $A_{y y}$ and $A_{x z}$ in elastic nd scattering at the incoming neutron lab. energy $E=135 \mathrm{MeV}$. For description of lines see Fig. 5. The pd data (open circles) are from ref. [24]

The $3 \mathrm{~N}$ Faddeev equation is set up for a breakup operator and solved in momentum space and partial wave projected. In the relativistic case Jacobi momenta are constructed using Lorentz boosts instead of Galilean boosts, the resolvents involve relativistic kinetic energies, the two-body interactions in the three-body problem appear inside of square roots in a manner dictated by S-matrix cluster properties, and the permutation operators include Wigner rotations [18-20].

When the 3NF's do not act, the effects of relativity are seen in Nd elastic scattering cross section at backward angles only (see Fig. 5). Relativity increases slightly the non-relativistic cross section. For spin observables only small effects of relativity are observed [18] (see Fig. 6). When a 3NF is included the interplay of relativity and 3NF's leads to a slight increase of cross sections at angles larger than $\theta_{c m} \approx 100^{\circ}$, again leading only to small effects for spin observables [20] (see Figs. 5, 6).

For the breakup cross section large relativistic effects are localized in specific regions of the phase-space. They lead to a characteristic pattern of relativistic versus non-relativistic cross section and at $E_{N}^{\text {lab }}=200 \mathrm{MeV}$ those changes can be up to $\approx \pm 60 \%$ [21]. For breakup spin observables effects of relativity found in calculations with NN forces only are seen with practically the same magnitude when 3NF is added [20].

\section{Summary}

The chiral $\mathrm{N}^{3} \mathrm{LO} 3 \mathrm{NF}$ is too weak to explain the low-energy $A_{y}$ puzzle. It also does not provide an explanation of discrepancies found for cross sections in the nn QFS and SST configurations of the low-energy nd breakup. Existing nd data seem not to exclude the possibility of two neutrons forming bound ${ }^{1} S_{0}$ state with binding energy of $\approx-100 \mathrm{keV}$. The existence of the dineutron could provide an explanation for the nn QFS cross section discrepancy. 
An exactly Poincaré invariant formulation of three-nucleon scattering using realistic interactions leads to significant changes of the breakup cross section at higher energies and in certain regions of phase space. For the elastic scattering cross sections the small changes are restricted to backward angles and practically no effects are seen for spin observables. Therefore the relativity is not responsible for large discrepancies found in elastic $\mathrm{Nd}$ scattering. They must originate from $3 \mathrm{NF}$ components, which become active at higher energies. Therefore we expect that 3NF's in all their complexity have to be taken into account in $3 \mathrm{~N}$ Faddeev calculations.

Acknowledgments This work was supported by the Polish National Science Center under Grant No. DEC-2011/01/B/ST2/00578. It was also partially supported by the European Community-Research Infrastructure Integrating Activity "Exciting Physics Of Strong Interactions" (acronym WP4 EPOS) under the Seventh Framework Programme of EU. The numerical calculations have been performed on the supercomputer cluster of the JSC, Jülich, Germany and Ohio Supercomputer Centre, USA (Project PAS0680).

Open Access This article is distributed under the terms of the Creative Commons Attribution License which permits any use, distribution, and reproduction in any medium, provided the original author(s) and the source are credited.

\section{References}

1. Epelbaum, E.: Few-nucleon forces and systems in chiral effective field theory. Prog. Part. Nucl. Phys. 57, 654-741 (2006)

2. Machleidt, R., Entem, D.R.: Chiral effective field theory and nuclear forces. Phys. Rep. 503, 1-75 (2011)

3. Bernard, V., et al.: Subleading contributions to the chiral three-nucleon force: long-range terms. Phys. Rev. C 77, 064004$064016(2008)$

4. Bernard, V., et al.: Subleading contributions to the chiral three-nucleon force. II. Short-range terms and relativistic corrections. Phys. Rev. C 84, 054001-054022 (2011)

5. Skibiński, R., et al.: Triton with long-range chiral ( $\left.\mathrm{N}^{3} \mathrm{LO}\right)$ three-nucleon forces. Phys. Rev. C 84, 054005-054014 (2011)

6. Tornow, W., et al.: The low-energy neutron-deuteron analyzing power and the ${ }^{3} P_{0,1,2}$ interactions of nucleon-nucleon potentials. Phys. Lett. B 257, 273-277 (1991)

7. Krebs, H., Gasparyan, A., Epelbaum, E.: Chiral three-nucleon force at N4LO: longest-range contributions. Phys. Rev. C 85, 054006-054020 (2012)

8. Setze, H.R., et al.: Verification of the space-star anomaly in nd breakup. Phys. Lett. B 388, 229-234 (1996)

9. Siepe, A., et al.: Neutron-proton and neutron-neutron quasifree scattering in the $\mathrm{n}$-d breakup reaction at $26 \mathrm{MeV}$. Phys. Rev. C 65, 034010-034016 (2002)

10. Witała, H., Glöckle, W.: On the discrepancies in the low-energy neutron-deuteron breakup. J. Phys. G Nucl. Part. Phys. 37, 064003-064013 (2010)

11. Witała, H., Glöckle, W.: The nn quasifree nd breakup cross section: Discrepancies with theory and implications for the ${ }^{1} S_{0}$ nn force. Phys. Rev. C 83, 034004-034011 (2011)

12. Witała, H., Glöckle, W.: Di-neutron and the three-nucleon continuum observables. Phys. Rev. C 85, 064003-064011 (2012)

13. Gonzales Trotter, D.E., et al.: Neutron-deuteron breakup experiment at $E_{n}=13 \mathrm{MeV}$ : determination of the ${ }^{1} S_{0}$ neutronneutron scattering length $a_{n n}$. Phys. Rev. C 73, 034001-034021 (2006)

14. Koori, N.: Energy spectra of breakup protons from the D(n,p)2n reaction at $14.1 \mathrm{MeV}$ neutrons. J. Phys. Soc. Jpn. 32, 306$315(1972)$

15. Haight, R.C., Grimes, S.M., Anderson, J.D.: Measurement of the proton spectrum from the ${ }^{2} \mathrm{H}(\mathrm{n}, \mathrm{p}) 2 \mathrm{n}$ reaction at $13.98 \mathrm{MeV}$ and the neutron-neutron scattering length. Phys. Rev. C 16, 97-106 (1977)

16. Shirato, S., Koori, N.: Measurements of the cross sections for $\mathrm{n}-\mathrm{d}$ elastic and inelastic scattering at $14.1 \mathrm{MeV}$. Nucl. Phys. A 120, 387-400 (1968)

17. Golak, J., et al.: Electron and photon scattering on three-nucleon bound states. Phys. Rep. 415, 89-205 (2005)

18. Witała, H., et al.: Relativistic effects in neutron-deuteron elastic scattering. Phys. Rev. C 71, 054001-054012 (2005)

19. Witała, H., et al.: Relativity and the low-energy nd $A_{y}$ puzzle. Phys. Rev. C 77, 034004-034016 (2008)

20. Witała, H., et al.: Three-nucleon force in relativistic three-nucleon Faddeev calculations. Phys. Rev. C 83, 044001$044020(2011)$

21. Skibiński, R., et al.: Relativistic effects in exclusive neutron-deuteron breakup. Eur. Phys. J. A 30, 369-380 (2006)

22. Hatanaka, K., et al.: Cross section and complete set of proton spin observables in pd elastic scattering at $250 \mathrm{MeV}$. Phys. Rev. C 66, 044002-044011 (2002)

23. Maeda, Y. et al.: Differential cross section and analyzing power measurements for nd elastic scattering at $248 \mathrm{MeV}$. Phys. Rev. C 76, 014004-014016 (2007)

24. Sekiguchi, K., et al.: Complete set of precise deuteron analyzing powers at intermediate energies: comparison with modern nuclear force predictions. Phys. Rev. C 65, 034003-034018 (2002) 